IV. Kritik im Krisenmodus 



\title{
Zeit der Pandemie, Zeit der harten Wissenschaften? Über einen fatalen Fehlschluss und die Perspektiven einer kritischen politischen Theorie der Pandemie
}

\author{
Oliver Flügel-Martinsen
}

\section{Einleitung}

Die Corona-Pandemie wirbelt nicht nur unseren Alltag und unsere Lebenswirklichkeit durcheinander, hat unabsehbare und massive Auswirkungen auf die sozio-ökonomische und mittelfristig womöglich auch auf die politische Situation in vielen Ländern, sondern sie beeinflusst zudem in starkem Maße wissenschaftliche Diskurse. War in der öffentlichen Wahrnehmung auch vor Corona ein mehrheitlich am naturwissenschaftlichen Modell orientiertes Wissenschaftsverständnis fraglos vorherrschend, so scheint dieses Verständnis mittlerweile geradezu selbstverständlich zu sein. Das bedeutet, wie die öffentliche Präsenz eines die Grenze zu obskuren Verschwörungstheorien häufig überschreitenden Diskurses der Wissenschaftsgegnerschaft zeigt, keineswegs, dass naturwissenschaftliche Erkenntnismethoden unhinterfragt akzeptiert werden. Aber wenn derzeit von Wissenschaft die Rede ist, dann scheint gar kein Zweifel daran zu bestehen, dass damit ganz selbstverständlich eine am Bild der hard sciences orientierte Wissenschaftlichkeit gemeint ist, während ein Pluralismus wissenschaftlicher Erkenntnismodelle noch nicht einmal in Betracht gezogen wird. Das ist in öffentlichen Diskussionen vor allem auch dann der Fall, wenn über die unmittelbar mit einschlägigen epidemiologischen oder virologischen Fragen befassten Disziplinen hinaus auch auf den Bedarf an anderen wissenschaftlichen Perspektiven hingewiesen wird. ${ }^{1}$ Zwar mögen - wenn es etwa um Entscheidungen wie die Öffnung von Schulen oder Kinderbetreuungseinrichtungen geht - dann auch bildungs- und erziehungswissenschaftliche, psychologische oder soziologische Einschätzungen gefragt sein. Aber die Rolle, die diesen Disziplinen zugewiesen wird, ist wiederum klar am naturwissenschaftlichen Modell einer positivistischen Tatsachen-

1 Wie es bspw. der in Deutschland rasch zu nationaler Bekanntheit gelangte Virologe Christian Drosten anerkennenswerterweise immer wieder tut. 
wissenschaft konturiert: Erwartet werden Erkenntnisse, die sich auf verallgemeinernde Tatsachenbeobachtungen stützen. Die anderen Disziplinen im genannten Beispiel die Sozialwissenschaften - zeichnen sich innerhalb dieses Anforderungsprofils nicht durch andere Denk- und Reflexionsformen, sondern nur durch andere Gegenstände aus.

Das ist angesichts der enormen Gefahren und Herausforderungen, die mit einer Pandemie einhergehen, durchaus eine nachvollziehbare politische und öffentliche Erwartungshaltung. Die entscheidende Frage ist aber, ob durch ein solch einseitiges Wissenschaftsverständnis, dem zufolge Wissenschaft beratungs-, besser noch implementierungsfähige Fakteninformationen generiert, nicht wesentliche Dimensionen kritischer Erkenntnis und Reflexion konstitutiv verdrängt und abgeschnitten werden. Gerade an diesen könnte aber angesichts der enormen Unsicherheit, mit der uns die Pandemiesituation konfrontiert, ein hoher Bedarf bestehen.

Kritische Erkenntnis ist eben nicht auf einen Dissens zwischen unterschiedlichen Forschungsrichtungen innerhalb des gleichen wissenschaftstheoretischen Paradigmas reduzierbar. Wenn sich, wie es in der deutschen Medienöffentlichkeit seit Ausbruch der Corona-Pandemie Anfang 2020 der Fall ist, eine Auseinandersetzung zwischen mehreren virologischen Standpunkten entspinnt - etwa dem Christian Drostens, der tendenziell für striktere Seuchenschutzmaßnahmen plädiert, und jenem seines die mediale Aufmerksamkeit offensiv und nicht immer reputationssichernd suchenden Bonner Gegenstücks Hendrik Streeck, der sich für das ausspricht, was häufig eine mutigere, also eine für mehr Lockerungen plädierende Haltung genannt wird -, dann kommt darin noch kein wissenschaftlicher Pluralismus im starken Sinne zum Ausdruck. Beide vertreten verschiedene Hypothesen, die jeweils andere Schlüsse nahelegen, aber beide bewegen sich fraglos innerhalb eines positivistisch-naturwissenschaftlichen Paradigmas.

Ist das in einer Lage wie der unsrigen aber vielleicht auch alternativlos? Wirkt die Corona-Pandemie womöglich sogar wie ein heilsamer Schock, der zu einer Konzentration auf das Wesentliche führt? Und erscheinen dann Perspektiven wie die - um exemplarisch mein eigenes theoretisches Selbstverständnis heranzuziehen - einer objektivitäts- und wahrheitsskeptischen, kritischen politischen Theorie als ein Luxus vergangener Zeiten, für den angesichts der drängenden Probleme der Gegenwart kein Platz mehr ist? Ändert Corona alles?

Ich möchte diese Fragen zunächst aus einer persönlichen Perspektive aufnehmen, von der aus ich dann aber schnell zu einigen weiterführenden Überlegungen kommen werde: Subjektiv hat mich die Corona-Situation, wie auch viele andere Kolleg*innen, mit denen ich im Austausch stehe, zu- 
nächst beinahe schockhaft mindestens an den Rand einer lähmenden Sinnkrise geführt. Ich hatte gerade noch vor dem Lockdown im Frühjahr 2020 ein (mittlerweile erschienenes) Buch zur radikalen Demokratietheorie in den Druck gegeben (Flügel-Martinsen 2020) und wollte mich an die Überarbeitung der ersten Fassung eines längeren Essays setzen, der sich mit den Perspektiven politischer Theorie als kritischer Zeitdiagnose beschäftigt. Diese Themen und Fragestellungen schienen plötzlich aber geradezu wie aus der Zeit gefallen. Die unmittelbare, mutmaßlich massenhafte Gefahr des Erkrankens und für viele auch des Sterbens schob in meiner subjektiven Selbstwahrnehmung meine Forschungsfragen brachial beiseite. In der öffentlichen ebenso wie in der privaten Wahrnehmung standen in der ersten Zeit, verstärkt durch schockierende Bilder aus bereits stärker betroffenen Ländern, basale Fragen wie die der Gesundheits- und Lebensmittelversorgung im Vordergrund. Entsprechend dominant war in der Folge, auch als sich abzeichnete, dass der ganz schwere Pandemieverlauf wenigstens in der ersten Welle in Deutschland wohl ausbleiben würde, die eingangs skizzierte Fixierung auf wissenschaftliche Perspektiven, die sich am naturwissenschaftlichen Erkenntnismodell ausrichten. Blieben die virologischen und die epidemiologischen Herausforderungen weiterhin akut, traten dann noch die vielfach harten und in ihren Konsequenzen unabsehbaren, möglicherweise strukturellen Folgen des Lockdowns und anderer seuchenhygienisch gebotener Beschränkungen des öffentlichen Lebens als Probleme hinzu.

Doch beides - die im engeren Sinne auf die Eindämmung der Pandemie bezogenen Fragen ebenso wie diejenigen, die sich auf die Folgen der Maßnahmen zur Corona-Bekämpfung beziehen - wird dramatisch unterkomplex reflektiert, wenn der Blickwinkel, wie es in der öffentlichen Diskussion in weiten Teilen der Fall ist, auf politisch artikulierte Bedarfe an zu erbringende wissenschaftliche Erkenntnisleistungen und einen Konflikt zwischen Wissenschaft und Wissenschaftsgegner*innen reduziert wird. Zumal auf der Seite der Wissenschaftsgegner*innen bislang vielfach eine obskure Mischung aus rechtspopulistischen bis rechtsextremen, verschwörungstheoretischen und, sich leider oft auch allzu bedenkenlos hinzugesellenden, esoteriknahen naturheilkundeaffinen Personenkreisen die öffentliche Wahrnehmung dominieren. Wie der Blick auf zahlreiche Problemfelder und Kontroversen zeigt, verändert Corona eben nicht alles, sondern verstärkt in vielerlei Hinsicht Spannungen und Ungerechtigkeitsstrukturen, die einer kritischen Reflexion bedürfen - eine solche kritische Perspektive nimmt allerdings bislang eine zu geringe Rolle in der wissenschaftlichen ebenso wie in der öffentlichen Auseinandersetzung ein. 
Ich möchte diese kritische Sichtweise in vier Schritten kurz entfalten. In einem ersten Schritt werde ich danach fragen, ob die Zeit kontingenztheoretischer Wissenschafts- und Wahrheitsskepsis, wie sie für meine eigenen politiktheoretischen Arbeiten maßgeblich ist, im Lichte der Herausforderungen durch die Corona-Krise abgelaufen ist. Dabei wird sich allerdings rasch zeigen, dass es starke Gründe dafür gibt, gerade unter den jetzigen Umständen ein objektivitäts- und wahrheitsskeptisches Denken wachzuhalten (I.). Das führt in einem zweiten Schritt dazu, eine kritische Reflexion wissenschaftlicher und gesellschaftlich-politischer Maßstäbe als eine Kernaufgabe kritischen Denkens zu begreifen, die derzeit allerdings fatal ins Hintertreffen geraten ist (II.). Drittens ist es nach meinem Eindruck unerlässlich, darauf hinzuweisen, dass eine solche kritische Reflexion die Beziehung zwischen geltenden Maßstäben und hegemonialen Sinnordnungen herausstellen muss (III.). In der Fluchtlinie dieser Überlegungen wird es schließlich viertens möglich, zwei Aufgaben einer kritischen politischen Theorie der Pandemie zu umreißen (IV.).

\section{Wissenschaftliche Objektivität: Corona und das Ende der Wabrheitsskepsis?}

Setzt das Virus der Wahrheitsskepsis ein Ende? In Anbetracht des Bedarfs nach harter, faktengestützter medizinischer und naturwissenschaftlicher Forschung, der sich angesichts der elementaren Herausforderungen durch die Corona-Pandemie ergibt, könnte sich dieser Eindruck leicht aufdrängen. Einschätzungen, die auch teils lange vor der Corona-Krise - oftmals polemisch zugespitzt - geäußert wurden (vgl. Boghossian 2013), dass es sich bei der Betonung von Kontingenz und daraus abgeleiteten wahrheitsskeptischen Überlegungen um Spielereien, ja, so war auch immer wieder zu hören, um verantwortungslose Spielereien handle, scheinen sich seit Ausbruch der Pandemie zu bestätigen. Öffentlichkeitswirksam verkündet wurde das Ende des Kontingenzdenkens in jüngerer Zeit schon angesichts einer anderen harten Herausforderung, der des (wieder)erstarkten Rechtsnationalismus - zu der es nicht nur nichts zu sagen, sondern die es, so der zugespitzte Vorwurf, sogar mit, wenn nicht gleich wesentlich zu verantworten habe (vgl. Hampe 2016). Und wer will, so ließen sich solche Argumentationen fortschreiben, heute, konfrontiert mit der nächsten harten Tatsache, schon ernsthaft daran zweifeln, dass es wissenschaftlich objektiv konstatierbare Fakten gibt, wenn Menschen an einer Viruserkrankung sterben?

Derlei Einschätzungen haben sich allerdings auch schon vor Corona aus einer teilweise geradezu karikaturhaften Verzeichnung wahrheitsskepti- 
scher Positionen gespeist. Aus der - etwa in Foucaults Arbeiten vertretenen - These, dass soziale und politische Verhältnisse ebenso wie wissenschaftliche Annahmen kontingent, also historisch wandelbar sind, und dass beide Dimensionen, die der sozialen und politischen Ordnungen und die der wissenschaftlichen Reflexion miteinander durch diskursive Machtbeziehungen verbunden sind, wird dann schnell die polemische Verzeichnung, es würde die bloße Beliebigkeit von allem und jedem postuliert, die keinerlei objektive Maßstäbe mehr zulasse (vgl. Boghossian 2013). In Foucaults Wissenschaftstheorie - um zur Illustration eine konkrete wahrheitsskeptische Position (vgl. Veyne 2008: Kap. III) heranzuziehen - geht es aber nicht darum, die Beliebigkeit von Sinnordnungen herauszustellen, sondern vielmehr darum, zu zeigen, dass es sich bei diesen Sinnordnungen immer auch um Machtordnungen handelt, um die Kämpfe ausgetragen werden. Diese Spannungsverhältnisse analysiert Foucault bekanntlich mit dem Begriff des Diskurses (vgl. Foucault 1971), dem die konflikthafte Dimension der Auseinandersetzung zwischen verschiedenen Wahrheiten konstitutiv eingeschrieben ist. Foucaults Wahrheitsskepsis führt nicht zur Behauptung der Beliebigkeit von Wahrheit, sondern zu deren historischer Situierung in Machtbeziehungen, die sich und damit bestimmte Regime der Veridiktion stabilisieren, die aber ebenso kontestiert werden können und deshalb, wie auch die Wahrheit, als wandelbar begriffen werden müssen. ${ }^{2}$

Pointiert ließe sich im Anschluss an solche Überlegungen festhalten, dass der Streit um die Wahrheit nicht im Medium einer durch Objektivität neutral agierenden wissenschaftlichen Auseinandersetzung ausgetragen werden kann, weil so etwas wie wissenschaftliche Objektivität selbst konstitutiv umstritten bleibt und weil dieser Streit vor allem nicht in einer von gesellschaftlichen Einflussnahmen abgeschirmten Sphäre der reinen Wissenschaft stattfindet, sondern selbst in Machtbeziehungen eingelassen ist. Die Wahrheits- und Objektivitätsskepsis bezieht sich dabei auch auf diese Beschreibung selbst: Sie kann nicht mit dem Argument höherer Einsicht als wahre Beschreibung der Entstehung und des Wandels von Wissensordnungen auftreten, sondern auch sie ist Teil einer anhaltenden Kontroverse. Kontroversen wie diese sind, wie Jacques Derrida in Die unbedingte Univer-

2 Vgl. zu Foucaults Exposition des Begriffs der Veridiktion seine Überlegungen zum Markt als Regime der Veridiktion in der Vorlesung am Collège de France vom 17. Januar 1979, die er dann sogleich auch auf andere diskursive Ordnungen ausweitet, Foucault (2004: 31-38). 
sität nachdrücklich herausstellt, wesentlich Bestandteil der vielfältigen Reflexionsbewegungen in den humanities (vgl. Derrida 2001).

Sicherlich besteht in Zeiten der Pandemie ein erhöhter Bedarf nach der medizinischen und naturwissenschaftlichen Erforschung des Virus, seiner Verbreitungswege und möglicher Bekämpfungsstrategien. Daraus aber die unhinterfragte Gültigkeit eines naturwissenschaftlichen Erkenntnismodells, das sich auf Objektivität und vermeintliche Tatsachenwahrheit stützt, abzuleiten, wäre fatal. Immer wieder zeigt sich gerade auch angesichts der oft widersprüchlichen Einschätzungen, die sich aus naturwissenschaftlichen Untersuchungen ableiten lassen, dass hier grundlegende Fragen berührt werden, die sich nicht einfach durch mehr Forschung klären lassen, sondern die häufig auch eine Kontroverse über die Maßstäbe umfassen (müssten), anhand derer diese Einschätzungen formuliert werden. Der von medizinisch Forschenden, die sich berechtigterweise nicht politisch zur Verantwortung ziehen lassen möchten, häufig geäußerte Hinweis, dass es eine Aufgabenteilung zwischen Wissenschaft und Politik gebe, der zufolge politische Akteur*innen verantwortliche Entscheidungen auch unter Einbeziehung anderer Gesichtspunkte als solchen rein wissenschaftlichen Typs treffen müssen, ist nachvollziehbar, beschreibt die Situation aber unterkomplex. Auch dass dann, wie etwa in Drostens zumeist sehr differenzierten Einlassungen der Fall, ein Bedarf nach der wissenschaftlichen Expertise anderer, etwa sozialwissenschaftlicher Disziplinen postuliert wird, ist vermutlich gut gemeint, trifft aber nicht den entscheidenden Punkt. Was vor allem erforderlich ist und innerhalb eines am Ideal objektiver Wissenschaft ausgerichteten Diskurses gar nicht erst erschlossen werden kann, ist eine kritische Reflexion der Beziehung von Maßstäben - der Objektivität, des Erforderlichen usf. - und hegemonialen Sinn- und Machtordnungen. Zu denken ist hier an Fragen wie beispielsweise die folgenden, auf die sich gerade keine Antworten im Sinne einer objektiven wissenschaftlichen Einschätzung geben lassen: Wer oder was wird aus welchen Gründen als systemrelevant verstanden und erfährt deshalb eine höhere Zuwendung von Ressourcen? Welche Aufgaben werden als private, welche als öffentliche verstanden und wer nimmt sich ihrer (z.B. der Kinderbetreuung) aufgrund der Fortdauer welcher Machtverhältnisse vor allem an? Wessen Leben wird aus welchen Gründen in welchem Umfang eingeschränkt? Oder auch nicht zuletzt: Wessen Leben wird aus welchen Gründen größeren Risiken ausgesetzt? ${ }^{3}$ - Fragen wie diese machen eine kriti-

3 Vgl. zur Frage der Vulnerabilität des Lebens und der asymmetrischen Verteilung der Risiken insgesamt Butler 2009. 
sche Reflexion der Beurteilungsmaßstäbe erforderlich, die sich wenigstens nicht allein - und ich würde dafürhalten: auch nicht primär - auf erfahrungswissenschaftlich gesicherte Erkenntnisse stützen kann.

\section{Eine kritische Reflexion von Beurteilungsmaßstäben}

Nietzsches Wissenschaftsphilosophie kulminiert in der wissenschaftsskeptischen These, dass Wissenschaft, die dem eigenen Anspruch nach Überzeugungen und Glauben durch Einsichten und objektive Beurteilungsmaßstäbe zu ersetzen sucht, in letzter Instanz contre coeur von den alten und lediglich überwunden geglaubten Bezugspunkten des Glaubens und der Überzeugungen heimgesucht werde (vgl. Nietzsche 1999: 577, s.a. Flügel-Martinsen 2011: 71ff.). Wissenschaft ist intern demnach gerade nicht wissenschaftlich verfasst, sondern folgt einer Glaubenslogik, die sie dem eigenen Anspruch zufolge gerade zu überwinden sucht. Eine solche Wissenschaftskritik ist in teils sehr unterschiedlichen Varianten seitdem zwar, prominent u.a. in den Arbeiten Foucaults (1971 und 1997) oder auch Habermas' (1969), ${ }^{4}$ immer wieder gegen das am naturwissenschaftlichen Ideal ausgerichtete Erkenntnismodell ins Feld geführt worden, aber für das naturwissenschaftliche Selbstverständnis im Grunde weitgehend folgenlos geblieben. Solange allerdings die Residuen des Glaubens in der als objektive Tatsachenanalyse auftretenden Wissenschaft ausgeblendet werden, versagt sich diese die erkenntnistheoretischen Mittel einer selbstkritischen Reflexion der eigenen Maßstäbe. Zwar ist eine Politik, die wissenschaftliche Einsichten aus instrumentellen und strategischen Gründen einfach beiseiteschiebt, wie es der Populismus à la Trump aggressiv und mit klaren strategischen Absichten zelebriert, fraglos unter die schlimmsten politischen Erscheinungen der Gegenwartswelt zu rechnen. Allerdings kann das adäquate Gegenprogramm auch nicht in einer Politik bestehen, die, wie es in der derzeitigen Corona-Krise vielfach der Fall ist, Wissenschaft als Expertiselieferantin einzuspannen sucht, um die Entscheidungen an vermeintlich neutralen und objektiven Maßstäben zu orientieren.

4 Wenn hier Habermas' und Foucaults Wissenschaftstheorie beinahe in einem Atemzug genannt werden, dann ist darauf hinzuweisen, dass beide sehr vieles trennt: Während Foucault eine wahrheitsskeptische Diskursanalyse und Genealogie wissenschaftlicher Rationalität schreibt, die selbst nicht mehr, jedenfalls nicht bruchlos, als rationale Wissenschaft aufzutreten beansprucht, dann dient Habermas' Kritik des naturwissenschaftlichen Erkenntnismodells demgegenüber in erster Linie der Verteidigung eines anderen Rationalitätsverständnisses. 
Unter anderem Hannah Arendt verdanken wir den Hinweis, dass Vorurteile, nach ihrem Verständnis also Urteile, die sich auf bereits verfügbare Maßstäbe stützen, nicht per se in allen Hinsichten problematisch, sondern vielmehr durchaus erforderlich sind, um subjektive und gemeinschaftliche Weltbeziehungen alltäglich vollziehen und organisieren zu können (vgl. Arendt 2003: 20ff.). Eine wichtige Überlegung Arendts ist es aber, dass von Urteilen, die im Modus des Vorurteils vollzogen werden, nur in begrenztem Umfang Gebrauch gemacht werden darf, soll deren ideologische Verselbständigung verhindert werden. Gerade in Zeiten der Krise, die immer auch Zeiten der Orientierungslosigkeit und damit des erhöhten Orientierungsbedarfs sind, droht sich die Tendenz zur Verselbständigung und Verfestigung von Vorurteilen in besonderem Maße Bahn zu brechen (vgl. Arendt 2003: 21). Arendts Ausführungen zu „Pseudotheorien, die als geschlossene Weltanschauungen oder alles erklärende Ideologien die gesamte geschichtliche und politische Wirklichkeit zu begreifen vorgeben“ (Arendt 2003: 21), lesen sich dabei übrigens streckenweise auch wie eine Analyse jener Verschwörungstheorien, die sich in den letzten Jahren einer erschreckenden Beliebtheit erfreuen - und sie zeigen, dass diese im Grunde die Form einer manisch gewordenen Überakzentuierung des objektivwissenschaftlichen Verfahrens der Subsumtion eines Besonderen unter ein Allgemeines annehmen: Wo das Urteil auf der Grundlage des Vorurteils aus pragmatischen Erkenntnisgründen nur ein Einzelnes, aber nicht noch einmal den Maßstab beurteilt, wird nun in Verschwörungstheorien eine vermeintlich Erklärung und Orientierung bietende Überzeugung völlig ungeprüft und unüberprüfbar zur arkanen Welterklärungsformel überhöht, in deren Licht den Eingeweihten alles klar werde, während die übrige Welt weiter im Dunkeln tappe.

Arendt schwebt nun als entscheidende Form des Urteilens in Situationen, in denen die Orientierungsmaßstäbe nicht länger zur Verfügung stehen - was nach ihrer Einschätzung unter Bedingungen der modernen Welt im Grunde der Regelfall ist (vgl. Arendt 2003: 22) - das maßstabslose Urteilen auf der Grundlage der Urteilskraft vor. Im Raum des Politischen, so können ihre Überlegungen in ihrem Buch Über die Revolution zu einer gemeinschaftlichen Konstitution der Welt verstanden werden, ist dafür die Etablierung einer öffentlichen Sphäre der Politik wesentlich, die sich nicht durch Probleme wie soziale Verteilungsfragen belastet sieht, die der freiheitlich weltgestaltenden Politik zum Verhängnis werden können, indem sie Dimensionen des Notwendigen und des Erforderlichen ins Spiel bringen (vgl. Arendt 2000: Kap. 2). Dies ist der Punkt, an dem es meines Erachtens auf der Suche nach einer Theorie der kritischen Reflexion von Maßstäben nicht länger sinnvoll erscheint, Arendts Überlegungen zu folgen. 
Wichtig bleibt nach meinem Dafürhalten ihre Einsicht, dass Maßstäbe nicht selbst wiederum auf der Grundlage von Maßstäben - wie, im vorliegenden gedanklichen Kontext, solchen der wissenschaftlichen Objektivität - kritisch reflektiert werden können, da ja auch diese zur prüfenden Disposition stehen. Allerdings haben die obigen Ausführungen zu Foucault bereits angedeutet, dass sich eine Sphäre der von sozialen Machtbeziehungen befreiten öffentlichen Reflexion gerade nicht abtrennen lässt, sondern der Zusammenhang zwischen Beurteilungsmaßstäben, also den normativen Gehalten von Sinnordnungen, und Machtkonstellationen kritisch ausgeleuchtet werden muss.

\section{Hegemoniale Ordnungen}

Die Einsicht, dass gerade auch alltägliche Überzeugungen und Wahrheiten Ausdruck von hegemonialen Ordnungen sind, hat mit einer großen, bis heute anhaltenden Wirkung der italienische Marxist Antonio Gramsci in den Diskurs kritischen Denkens eingespeist. Bei Gramsci steht diese auch für eine kritische Wissenschafts- und Rationalitätstheorie wichtige Überlegung in dem sehr praktischen Erkenntniszusammenhang der Suche nach geeigneten revolutionären Strategien. Gegen die in marxistischen Diskursen verbreitete Tendenz, Politik, Kultur und Wissenschaft zu bloßen Epiphänomenen, ja teils nur zu Reflexen einer materiellen Basis zu erklären, ist Gramsci auf der Suche nach einer erfolgreichen revolutionären Strategie auf die Bedeutung von weithin geteilten Überzeugungen und Wahrheiten gestoßen. In ihnen bringt sich - so der Kern jener Hegemonietheorie, die dank der zentralen Stellung, die ihr Laclau und Mouffe in $\mathrm{He}$ gemonie und radikale Demokratie (vgl. 2012) gegeben haben, in zeitgenössischen Bemühungen um eine kritische politische Theorie eine prominente Rolle spielt - auf eine gleichsam subkutane und deshalb nicht auf Anhieb sichtbare Weise die hegemoniale Ordnung zum Ausdruck. Oder vielmehr anders herum: Eine Ordnung erweist sich dann als hegemonial, wenn die für sie maßgeblichen Überzeugungen zum Alltagsverstand - zum Common Sense - geworden sind. Chantal Mouffe hat so jüngst noch einmal den Erfolg des neoliberalen Diskurses am Beispiel der Thatcherschen Politik auch über deren Regierungszeit hinaus analysiert, indem sie darlegt, dass die zentrale Vision des Neoliberalismus im Großbritannien der 1990er so „tief im Common Sense verankert [war], dass die Labour Party, als sie 1997 mit Tony Blair wieder an die Macht kam, nicht einmal versuchte, die neoliberale Hegemonie in Frage zu stellen“ (Mouffe 2018: 43). 
Gleiches gilt, wie Michel Foucault mithilfe einer Analyse der Ordnung des Diskures für Wissensordnungen gezeigt hat, auch für wissenschaftliche Wahrheiten, Methodologien und Überzeugungssysteme: Sie als diskursive Ordnungen zu analysieren, heißt, sie als Ausdruck stabilisierter Machtbeziehungen zu begreifen. Am wirksamsten sind solche Ordnungen, diese Überlegung teilen Gramsci und Foucault, wenn die grundlegenden Überzeugungen geradezu selbstverständlich sind, also gar nicht mehr in Frage gestellt werden. Das ist allerdings nur selten der Fall. In der Regel haben wir es mit einem komplexen Gefüge von Diskursen und Gegendiskursen zu tun. In der am Hegemoniebegriff orientierten Theoriesprache Laclaus und Mouffes handelt es sich dabei um konkurrierende hegemoniale Projekte (vgl. Laclau/Mouffe 2012).

Um die Persistenz einer bestehenden Hegemonie zu verstehen, ist Gramscis Verweis auf den Alltagsverstand wesentlich: Was auf diese Weise für wahr gehalten wird, wird nämlich einer kritischen Reflexion in der Regel gar nicht erst ausgesetzt, schlicht weil es als selbstverständlich gilt. Jede ernsthafte kritische Reflexion von Maßstäben muss demnach diese gleichsam unsichtbare wirksame Schutzmembran hegemonialer Wahrheiten durchbrechen, um herauszustellen, dass gerade auch selbstverständlich Wirkendes ein Ausdruck bestehender Machtverhältnisse ist. Eine solche kritische Analyse kann aber - das ist in unserem Zusammenhang der entscheidende Punkt - nicht innerhalb der etablierten Orientierungsrahmen wissenschaftlicher oder politischer Verantwortung erfolgen, weil sie ja gerade darauf angewiesen ist, über diese Orientierungsrahmen hinauszudenken.

Nicht alle über das Bestehende hinausgehenden Gegendiskurse sind kritische Analysen oder Bewegungen in irgendeinem emanzipatorisch zu nennenden Sinne. Die schon länger anhaltenden Infragestellungen der liberalen Demokratie durch eine Allianz von Rechtspopulist*innen bis hin zu Rechtsextremen sind politische Gegendiskurse - und auch die vielfach verschwörungstheoretische Anti-Corona-Bewegung, die vor allem in Deutschland Zulauf erfahren hat, ist eine gegenhegemoniale Bewegung zur Hegemonie des naturwissenschaftlichen Paradigmas. Gegenhegemonial zu agieren, ist für sich allein genommen demnach keineswegs per se in irgendeiner Hinsicht emanzipatorisch. Und die bloße Eigenschaft, eine bestehende Ordnung zu kontestieren, kann deshalb auch nicht, wie es insbesondere gerne von rechts reklamiert wird, als Legitimation des eigenen, häufig mit menschenfeindlicher Gewalt in Wort oder Tat verbundenen Tuns beansprucht werden. Das heißt aber auch keineswegs, dass sich nichts dazu sagen lässt, welche Infragestellungen als emanzipatorische Kritiken zu verstehen sind und welche nicht. Abschließend möchte ich diese 
wichtige Frage noch etwas weiterverfolgen, indem ich zwei Aufgaben einer kritischen politischen Theorie der Pandemie umreiße.

\section{Zwei Aufgaben einer kritischen politischen Theorie der Pandemie}

Was also wären mögliche Aufgaben einer kritischen politischen Theorie der Pandemie? In den vorangegangenen Überlegungen hat sich gezeigt, dass eine solche kritische politische Theorie ihren Ort nicht innerhalb eines am naturwissenschaftlichen Erkenntnismodell orientierten Wissenschaftsverständnis finden kann. Vielmehr muss sie auch Erkenntnismodelle, Wahrheitsbegriffe und Beurteilungsmaßstäbe einer kritischen Befragung unterziehen, die sich schon allein deshalb nicht innerhalb eines vorab abgesteckten wissenschaftlichen Rahmens bewegen kann, weil sie ja nicht zuletzt dessen Beziehung zu hegemonialen gesellschaftlichen Ordnungen untersuchen muss.

Damit tritt bereits die erste Aufgabe hervor, die eine kritische politische Theorie im Kontext der Pandemie übernehmen kann: Sie ist eine kritische Begleitung gegenwärtiger Entwicklungen, Entscheidungen und Beurteilungsmaßstäbe, die im Zuge dieser Reflexion über scheinbar selbstverständliche Überzeugungen und Wahrheiten hinausdenkt, indem sie zeigt, wie diese auf politische Weise als Folge erfolgreicher hegemonialer politischer Projekte in die Welt gebracht werden. Gleichzeitig macht sich eine solche kritische politische Theorie aber auch reflexiv selbst zum Gegenstand und stellt ihr eigenes Tun in Frage - um ernsthaft als kritisch gelten zu können, muss sie immer auch eine selbstreflexive Kritik umfassen. Das ist auch ein markanter Unterschied zu jenen Verschwörungstheorien, die heute gerne einen starken Widerhall in rechten Bewegungen finden: Diese präsentieren sich zwar mit großem Pathos als aufrechter Widerstand und kritische Reflexion - dass sie das aber keineswegs sind, zeigt sich schon am bloßen Umstand, dass sie sich und ihre eigenen Maßstäbe nicht nur nicht in Frage zu stellen bereit sind, sondern jeglicher Infragestellung geradezu entziehen, indem sie behaupten, auf unverbrüchliche Wahrheiten zugreifen zu können. Sei es das angeblich bessere Wissen, das die geheimen $\mathrm{Zu}$ sammenhänge aufdecken soll, oder seien es die aggressiv und xenophob beanspruchten angeblichen Rechte eines nationalistisch verstandenen Volkes - stets sind es selbst nicht mehr befragbare Bezugspunkte, die in Anspruch genommen werden. Zudem erfolgen diese rechten Infragestellungen im Namen einer Inanspruchnahme von Rechten, die anderen vorenthalten werden sollen, während die kritische Perspektive, der ich hier nachspüre, Machtasymmetrien generell einer unnachgiebigen Infragestellung 
unterzieht und nicht auf die Fixierung von Exklusionen oder die Verteidigung von Privilegien zielt.

Besteht die erste Aufgabe demnach in einer kritischen Reflexion von selbstverständlich in Anspruch Genommenem, das auf seine Situierung in Machtbeziehungen hin befragt wird und dem so der Anschein des Selbstverständlichen abgestreift werden kann, so ist die zweite Aufgabe, die sich in den letzten Überlegungen bereits angedeutet hat, diagnostischer Art, indem es nun die Folgen der Asymmetrien dieser Machtbeziehungen sind, die in den Fokus gerückt werden. Auch in der medialen Berichterstattung ist zu Recht vielfach darauf hingewiesen worden, dass sich in der CoronaKrise Asymmetrien verstärken oder alte Asymmetrien wieder revitalisiert werden. $\mathrm{Zu}$ denken ist dabei etwa an das Beispiel der afroamerikanischen Bevölkerung in den USA, die in vielerlei Hinsicht stärker von der Pandemie betroffen ist, da sie aufgrund ihrer sozioökonomischen Stellung mehr und stärkere Erkrankungen, daher auch mehr Todesfälle zu beklagen hat und, mit Blick auf die Konsequenzen der Pandemiebekämpfung, höher von Arbeitslosigkeit betroffen ist. In Deutschland ließ sich in der Situation des Lockdowns im Frühjahr 2020 und der auch über ihn hinaus anhaltenden Schließung von Kinderbetreuungseinrichtungen und Schulen die Rückkehr zur alten, offensichtlich vorschnell überwunden geglaubten Praxis der Privatisierung von Elternlasten beobachten, die häufig stärker zu Lasten von Frauen ging - als besonders eindrückliche Gruppe sei hier nur auf die mehrheitlich weiblichen Alleinerziehenden verwiesen, die mit den Folgen der Schließung im Wortsinn allein gelassen wurden.

Mag Theorie auch, wie Hegel einmal über die Erkenntnisperspektive der Philosophie festhielt, darauf angewiesen sein, dass sich eine Entwicklung bereits vollzogen hat, um erkannt werden zu können, „da die Eule der Minerva [...] erst mit der einbrechenden Dämmerung ihren Flug“ (Hegel 1986: 28) beginnt, so lassen sich dennoch Entwicklungstendenzen kritisch beobachten. Im Falle der Corona-Pandemie bedarf es jedenfalls keiner hellseherischen Fähigkeiten, um zu der Einschätzung zu gelangen, dass Corona strukturelle Asymmetrien und die mit ihnen einhergehenden Ungerechtigkeiten verstärken wird. Konnte es zu Beginn der Pandemie daher noch so scheinen, als würden auch die Forschungsperspektiven kritischer politischer Theorie nun auf unerwartbare Weise neu ausgerichtet werden müssen, so zeigt sich in ihrem Fortgang zunehmend, dass Themen, deren sich kontingenztheoretische Ansätze kritischen politischen Denkens mit großem Nachdruck annehmen - wie etwa der wiedererstarkte Nationalismus, die Exklusion von Anderen, strukturelle Ungerechtigkeiten, Armut und sozioökonomische Prekarisierung, Homophobie oder pa- 
triarchale Heteronormativität - auch im Kontext der Pandemie aktuell bleiben und einer kritischen Reflexion bedürfen.

\section{Literaturverzeichnis}

Arendt, Hannah. 2000. Über die Revolution. München: Piper.

Arendt, Hannah. 2003. Was ist Politik? München: Piper.

Boghossian, Paul. 2013. Angst vor der Wahrheit. Berlin: Suhrkamp.

Butler, Judith. 2009. Frames of War. London/New York: Verso.

Derrida, Jacques. 2001. Die unbedingte Universität. Frankfurt/M.: Suhrkamp.

Flügel-Martinsen, Oliver. 2011. Jenseits von Glauben und Wissen. Bielefeld: transcript.

Foucault, Michel. 1971. L'ordre du discours. Paris: Gallimard.

Foucault, Michel. 1997. Archäologie des Wissens. Frankfurt/M.: Suhrkamp.

Foucault, Michel. 2004. Naissance de la biopolitique. Paris: Gallimard/Seuil.

Habermas, Jürgen. 1969. Technik und Wissenschaft als Ideologie. Frankfurt/M.: Suhrkamp.

Hampe, Michael. 2016. Katerstimmung bei den pubertären Theoretikern. DIE ZEIT. https://www.zeit.de/2016/52/kulturwissenschaft-theorie-die-linke-donald-t rump-postfaktisch-rechtspopulismus.

Hegel, G.W.F. 1986. Grundlinien der Philosophie des Rechts. Werke, Bd. 7. Frankfurt/M.: Suhrkamp.

Laclau, Ernesto/Mouffe, Chantal. 2012. Hegemonie und radikale Demokratie. Wien: Passagen.

Mouffe, Chantal. 2018. Für einen linken Populismus. Berlin: Suhrkamp.

Nietzsche, Friedrich. 1999. Die fröbliche Wissenschaft, KSA 3. München/Berlin/New York: dtv/de Gruyter, 343-651.

Veyne, Paul. 2008. Foucault. Sa pensée, sa personne. Paris: Albin Michel. 
